\title{
UTILIZACIÓN DE LOS HOSPITALES PÚBLICOS POR LOS EXTRANJEROS EN LA PROVINCIA DE MÁLAGA ${ }^{1}$
}

\author{
Carmen Carvajal Gutiérrez \\ Juan Corpas Alba
}

\begin{abstract}
"Tras la conquista de Málaga por las tropas de los Reyes Católicos se formó en el suelo malagueño una sociedad donde convivían las tres religiones monoteístas que imperaban en el siglo XVI: cristiana, islámica y judaica."

Ma Isabel Pérez de Colosía (1987)
\end{abstract}

\begin{abstract}
RESUMEN
Para conocer la utilización de los hospitales públicos por los extranjeros se han podido conseguir los datos sobre asistencia desde el año 2.000 hasta el año 2.004 en los hospitales Virgen de la Victoria, Costa del Sol, Antequera y Carlos Haya. Los resultados obtenidos muestran que se ha producido un fuerte crecimiento en todos los tipos de asistencia hospitalaria a extranjeros pero inferior al crecimiento de la población extranjera residente en la provincia de Málaga, y también muestran un uso diferente de la asistencia hospitalaria por parte de los extranjeros procedentes de la Unión Europea respecto de los extranjeros no comunitarios.
\end{abstract}

\section{ABSTRAT}

In order to know the use of public hospitals by foreigners we have been able to get data on assistance from 2.000 to 2.004 from the hospitals Vírgen de la Victoria, Costa del Sol, Antequera and Carlos Haya. The results show the growth of all types of hospital assistance to foreigners, although this increase has been less than that of foreign population living in the province of Málaga resides, the results illustrate the different use that population from the European Union make of hospital services with regards to non U.E. foreigners.

1. Este artículo forma parte de un proyecto sobre la utilización de los servicios públicos por los extranjeros en la provincia de Málaga, financiado por la D. G. de Coordinación de Políticas Migratorias de la Junta de Andalucía. 


\section{EL MARCO GENERAL LEGAL DE LA ASISTENCIA SANITARIAA EXTRANJEROS}

La Ley orgánica 4/2.000 de 11 de enero sobre derechos y libertades de los extranjeros en España y su integración social, en su artículo 12 delimita de forma general el derecho a la asistencia sanitaria de estos colectivos según el cual los extranjeros que se encuentren empadronados en España y los menores de dieciocho años, tienen derecho a la asistencia sanitaria en las mismas condiciones que los españoles; así como las extranjeras embarazadas tienen derecho a la asistencia en el embarazo, parto y posparto. Todos los demás extranjeros que se encuentren en España tienen derecho a la asistencia sanitaria pública de urgencias.

El elemento clave que garantiza el acceso a la asistencia sanitaria pública de los inmigrantes extranjeros es estar empadronado y haber tramitado la tarjeta sanitaria individual, documento de reconocimiento temporal a la asistencia sanitaria que los adscribe a un determinado Centro o Distrito de Salud; tiene una validez de un año y es renovable hasta que la persona cambie su situación.

Este marco general es matizado por los acuerdos en materia de prestaciones sanitarias de los países miembros de la Unión Europea que son básicamente unos acuerdos de devolución del costo de la asistencia sanitaria realizada a aquellos ciudadanos cubiertos por la sanidad pública de su país que vienen provistos del documento E111.

Dada la importancia de este tema ha sido abordado por las administraciones autonómicas que tienen delegadas las competencias sanitarias y en el caso de la Comunidad Autónoma de Andalucía tanto en el I Plan Integral para la inmigración (2001-2004), como en el II Plan Integral para la Inmigración (2006-2009) aprobado por el Parlamento andaluz el 9 de mayo, entre las líneas de actuación priorizadas se encuentra asegurar el acceso en condiciones de igualdad de la población inmigrante a los servicios sanitarios. Una de las novedades de este II Plan Integral para la Inmigración ha sido la descentralización por provincias para así adecuarse a las características peculiares de la inmigración en los distintos escenarios andaluces por lo que en el proyecto de Plan Integral para la Inmigración de la provincia de Málaga se consideran objetivos específicos facilitar el acceso al Servicio Sanitario Público a la población inmigrante, facilitar la adaptación de la atención sanitaria a las necesidades específicas de los extranjeros por sus características culturales, sociales o personales, así como formar a los profesionales de la salud y reducir el riesgo a la salud de la exclusión social más vulnerable entre los extranjeros. 


\section{ESCASEZ DE ESTUDIOS ESPECÍFICOS}

Dada la importancia económica y social de la atención sanitaria a los extranjeros, cuando la actual ley 4/2000 estaba en trámite de elaboración lógicamente se hicieron estudios para evaluar el posible coste económico de la universalización de esta asistencia sanitaria a los extranjeros en España (Ministerio de Trabajo 1999). Esta evaluación previa debía continuarse con los estudios sobre el uso que llevan a cabo estos extranjeros de los servicios sanitarios puesto que se trata de unos colectivos peculiares por su composición por edad y sexo, por su situación laboral y personal, etc. Así lo manifiestan el Consejo de Europa (2000) y los grupos de expertos de Naciones Unidas (Carballo, 2005): los inmigrantes están especialmente expuestos a problemas de salud, muchos proceden de entornos socioeconómicos desfavorecidos con limitado acceso a la atención sanitaria, y además tienden a dirigirse a entornos sociales y económicos que siguen exponiéndoles a enfermedades ligadas a la pobreza. A esto se une que en los primeros momentos de la inmigración por la inseguridad del empleo, el trastorno de la vida familiar, etc. están más predispuestos que los autóctonos a desarrollar problemas sicosomáticos y de enfermedad mental; los niños también sufren esta situación de estrés y la insuficiente presencia de los padres por sus horarios laborales. Finalmente se ha constatado que los trabajadores extranjeros presentan mayores tasas de accidentes laborales.

Por todo ello la Asamblea Parlamentaria del Consejo de Europa (2000) recomienda entre otros aspectos que se favorezca el sistema de estadísticas relativas a la atención sanitaria dispensada a inmigrantes y conocer los obstáculos que puedan encontrar en el acceso a la atención sanitaria.

Recogiendo esta preocupación en el $4^{\circ}$ Congreso Sobre la Inmigración en España, celebrado en Girona en noviembre de 2004, contó con una mesa sobre Migraciones y Salud, y la mayor parte de las ponencias y comunicaciones señalan y justifican la necesidad de hacer estudios; sin embargo hasta ahora se han podido acometer fundamentalmente estudios generales o colaterales en torno al tema bien como uno de los pilares del estado de bienestar (R. Aparicio y A. Tornos, 2005), o haciendo análisis comparados entre la diferente atención socio sanitaria por países (Moreno, 2004: Alcalde y otros, 2004), o se refieren a problemáticas específicas de salud mental (Lurbe, 2004). En muy pocos estudios se ha acometido la medición directa de la asistencia sanitaria a extranjeros y se ha hecho tratándolo como un colectivo homogéneo, sin diferenciar por nacionalidad (I. y L. Aparicio, y C. P. Cuevas, 2004).

Como sabemos dentro de la comunidad autónoma andaluza, Málaga es la provincia con mayor número de extranjeros residentes y con un fuerte crecimiento, confluyendo dos tipos de migración internacional muy dispares: los jubilados del norte y centro de Europa, y los inmigrantes económicos básica- 
mente no comunitarios. Estos extranjeros están presentes en toda la provincia pero sobre todo en la Costa del Sol y en la capital. En estas zonas las previsiones efectuadas sobre necesidades sanitarias de la población se pueden ver totalmente desbordadas por la inmigración.

En esta provincia de Málaga se han efectuado estudios muy importantes que se interesan por la asistencia sanitaria a los extranjeros pero que no los abarcan en su totalidad: se han tenido en cuenta bien solo a los mayores comunitarios sobre todo británicos (Beta y Caia, 1998; Echezarreta, 2005; Rodríguez y Warners, 2002) o a las poblaciones flotantes de turistas (Hospital Costa del Sol, 2002).

Por todo ello estamos tratando de conocer que tipo de asistencia sanitaria pública están requiriendo los extranjeros en la provincia de Málaga según nacionalidad. Ya abordamos en una investigación anterior el uso de la atención primaria (Carvajal y Corpas, 2006); en este artículo vamos a tratar de acercarnos al análisis de la atención hospitalaria.

\section{PROBLEMA DEL ACCESO A LOS DATOS}

El primer paso que acometimos fue ponernos en contacto con los servicios centrales del SAS (Servicio Andaluz de Salud) para solicitar esta información; este servicio tuvo una respuesta perfectamente razonada que justificaba la escasez de datos centralizados disponibles sobre la utilización de los servicios sanitarios por los extranjeros; datos que vamos a reproducir pues al ser tan limitados validan los resultados obtenidos en nuestra investigación como una auténtica aportación.

Puesto que no estaba centralizada esta información, nos hemos puesto en contacto con cada uno de los seis centros hospitalarios solicitando la información sobre los usuarios extranjeros. El problema es que la nacionalidad del usuario en muchos casos no se declara o se hace incorrectamente y cada centro hospitalario ha tenido su propio sistema de recogida y tratamiento de la información por lo que los datos que hemos podido obtener son muy desiguales:

- En el hospital Virgen de la Victoria, con categoría de hospital de especialidades, ubicado en la capital y que atiende a la zona hospitalaria Centro, nos ofrecieron listados no informatizados de las urgencias, consultas, ingresos e intervenciones desde el año 2000 al 2004 inclusive, lo que nos ha supuesto un ingente trabajo de selección, depuración y elaboración de la información pues en lugar de la nacionalidad se declaraba en muchos casos la ciudad de nacimiento, o el continente; a pesar de esa labor de indagación directa ha quedado un margen de nacionalidad desconocida en urgencias e ingresos que mantenemos en estadísticas como posible asistencia a extranjeros. 


\section{CUADRO 1}

\section{DATOS DISPONIBLES SOBRE ASISTENCIA A EXTRANJEROS EN LOS HOSPITALES PÚBLICOS DE LA PROVINCIA DE MÁLAGA ANTES DE NUESTRA INVESTIGACIÓN}

\begin{tabular}{|l|c|c|c|c|}
\hline Hospital & Año & Urgencias & Consultas & Ingresos \\
\hline \multirow{3}{*}{ Virgen de la Victoria } & 2002 & 35 & 3 & 2 \\
\cline { 2 - 5 } & 2003 & 35 & 46 & 6 \\
\hline \multirow{3}{*}{ Carlos Haya } & 2002 & 0 & 0 & 0 \\
\cline { 2 - 5 } & 2003 & 73 & 20 & 0 \\
\hline \multirow{3}{*}{ Costa del Sol } & 2002 & 96 & 167 & 16 \\
\cline { 2 - 5 } & 2003 & 2.080 & 4.113 & 500 \\
\hline \multirow{3}{*}{ Antequera } & 2002 & 12 & 14 & 5 \\
\hline \multirow{3}{*}{ Axarquía } & 2003 & 286 & 213 & 46 \\
\hline \multirow{2}{*}{ Serranía } & 2002 & 54 & 0 & 0 \\
\cline { 2 - 5 } & 2003 & 355 & 102 & 19 \\
\hline \multirow{2}{*}{ TOTAL } & 2002 & 0 & 0 & 0 \\
\cline { 2 - 5 } & 2003 & 61 & 80 & 14 \\
\cline { 2 - 5 } & 2002 & 197 & 184 & 23 \\
\hline
\end{tabular}

Fuente: Servicio de Planificación operativa S. A. S.

- Si nos pudieron ofrecer datos elaborados pero con muy desigual nivel de información en el Hospital Comarcal I Costa del Sol, ubicado en Marbella y que atiende a la Costa del Sol occidental; en el Hospital Regional Carlos Haya ubicado en la capital y en el Hospital Comarcal II de Antequera que atiende a la zona norte.

- Los hospitales comarcales de la Axarquía y Serranía, ubicados en Vélez Málaga y Ronda, a pesar de los esfuerzos de sus responsables no han podido individualizar a los usuarios extranjeros en sus bases de datos.

Lamentamos esta última carencia pero al haber podido obtener la información de los hospitales Costa del Sol, Virgen de la Victoria y Carlos Haya creemos que hemos podido medir la utilización fundamental de los servicios hospitalarios porque estos últimos son los hospitales públicos de mayor categoría en la provincia, es decir acogen todas las especialidades, y sobre todo porque estos hospitales se encuentran y atienden las zonas con mayor presencia de extranjeros residentes. Por todo ello los resultados que vamos a obtener en esta investigación respecto a utilización de los servicios públicos hospita- 
larios por los extranjeros en la provincia de Málaga no son concluyentes pero van a suponer un gran avance en su conocimiento.

Otras dificultades que entrañan estos datos se refieren a su tratamiento. En primer lugar porque se trata de tipos de asistencias muy diferentes que nunca podemos agregar lo cual nos obliga a una presentación poco sintética, pero además en una provincia turística como Málaga que cuenta con una gran cantidad de población flotante podemos hacer apreciaciones sobre intensidad de uso poniendo en relación las asistencias con la población extranjera empadronada pero de forma cualitativa, no mediante tasas e índices exactos puesto que cualquier asistencia médica especializada puede ser requerida por extranjeros no residentes.

\section{CRECIMIENTO DE LA ASISTENCIA A EXTRANJEROS EN LOS HOSPITALES PÚBLICOS DE MÁLAGA}

En los siguientes cuadros tenemos cuantificada la asistencia hospitalaria de urgencias, consultas e intervenciones recibida por los extranjeros. En ellos es fácil comprobar que en todos los hospitales de los que tenemos una serie de años se ha producido un fuerte incremento en todo tipo de asistencia a extranjeros:

\section{CUADRO 2}

ASISTENCIA A EXTRANJEROS EN URGENCIAS HOSPITALARIAS

\begin{tabular}{|c|c|c|c|c|}
\hline Año & $\begin{array}{c}\text { Hospital Virgen } \\
\text { de la Victoria }\end{array}$ & $\begin{array}{c}\text { Hospital Costa } \\
\text { del Sol }\end{array}$ & $\begin{array}{c}\text { Hospital de } \\
\text { Antequera }\end{array}$ & $\begin{array}{c}\text { Hospital Regional } \\
\text { Carlos Haya }\end{array}$ \\
\hline 2000 & 4.946 & & 91 & \\
\hline 2001 & 6.019 & 20.501 & 101 & \\
\hline 2002 & 7.779 & 24.473 & 140 & \\
\hline 2003 & 11.094 & 26.536 & 187 & \\
\hline 2004 & 12.908 & 30.010 & 231 & 5.934 \\
\hline
\end{tabular}

Fuente: Servicios de Documentación. Elaboración propia 


\section{CUADRO 3 ASISTENCIA A EXTRANJEROS EN CONSULTAS MÉDICAS}

\begin{tabular}{|c|c|c|c|c|}
\hline Año & $\begin{array}{c}\text { Hospital Virgen } \\
\text { de la Victoria }\end{array}$ & $\begin{array}{c}\text { Hospital Costa } \\
\text { del Sol }\end{array}$ & $\begin{array}{c}\text { Hospital de } \\
\text { Antequera }\end{array}$ & $\begin{array}{c}\text { Hospital Regional } \\
\text { Carlos Haya }\end{array}$ \\
\hline 2000 & 4.309 & & 30 & \\
\hline 2001 & 5.147 & 15.140 & 36 & \\
\hline 2002 & 6.571 & 19.802 & 92 & \\
\hline 2003 & 8.531 & 24.762 & 290 & \\
\hline 2004 & 10.231 & 30.553 & 469 & 4.563 \\
\hline
\end{tabular}

Fuente: Servicios de Documentación. Elaboración propia

\section{CUADRO 4}

\section{ASISTENCIA A EXTRANJEROS EN INGRESOS HOSPITALARIOS}

\begin{tabular}{|c|c|c|c|c|}
\hline Año & $\begin{array}{c}\text { Hospital Virgen } \\
\text { de la Victoria }\end{array}$ & $\begin{array}{c}\text { Hospital Costa } \\
\text { del Sol }\end{array}$ & $\begin{array}{c}\text { Hospital de } \\
\text { Antequera }\end{array}$ & $\begin{array}{c}\text { Hospital Regional } \\
\text { Carlos Haya }\end{array}$ \\
\hline 2000 & 1.486 & & 9 & \\
\hline 2001 & 1.581 & 2.716 & 12 & \\
\hline 2002 & 1.753 & 3.461 & 22 & \\
\hline 2003 & 1.990 & 3.840 & 33 & \\
\hline 2004 & 2.302 & 4.292 & 38 & 1.492 \\
\hline
\end{tabular}

Fuente: Servicios de Documentación. Elaboración propia

No obstante este crecimiento de la asistencia especializada a extranjeros ha sido inferior al ritmo de crecimiento de la población extranjera empadronada: por ejemplo si sumamos los tres hospitales de los que tenemos datos de 2001 a 2004, es decir Virgen de la Victoria, Costa del Sol y Antequera, las urgencias han pasado de 26.621 en 2001 a 43.149 en 2004, han crecido un 62\%; con el mismo procedimiento comprobamos que las consultas han crecido un $103 \%$ y los ingresos un 53,9\%. Sin embargo la población extranjera empadronada en la provincia de Málaga pasó de 85.480 empadronados a 1 de enero de 2001, a 181.589 a 1 de enero de 2005, es decir experimentó en estos años un crecimiento del $112,4 \%$. Podemos pues apuntar que todos los tipos de asistencia hospitalaria a extranjeros han crecido pero a un ritmo de crecimiento inferior al experimentado por el volumen de población extranjera residente. 


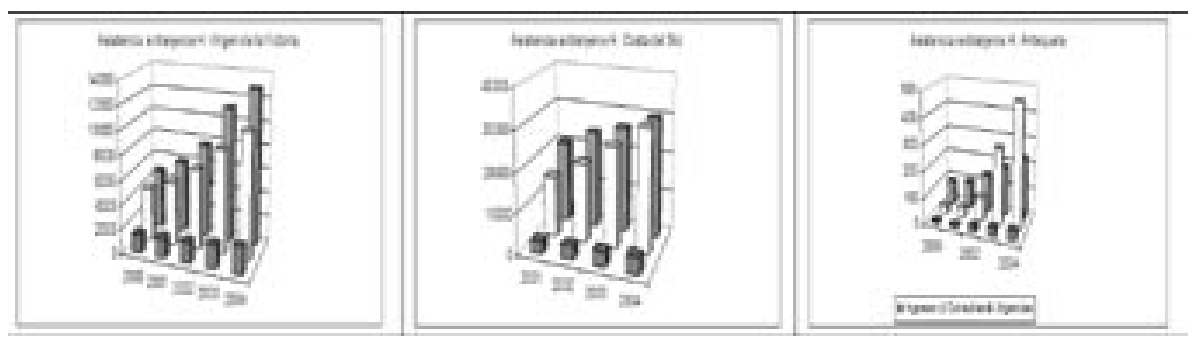

Fuente: Servicios de Documentación. Elaboración propia

No obstante hay diferencias entre unos centros y otros, y el gráfico anterior nos ayuda a comprobarlo: en todos los centros el ritmo de incremento más bajo se refiere a los ingresos hospitalarios, sin embargo mientras en los hospitales comarcales Costa del Sol y de Antequera el mayor crecimiento lo han experimentado las consultas de especialistas, en el Virgen de la Victoria lo han sido las urgencias. Esto puede deberse a la diferente categoría de estos hospitales: el hospital Costa del Sol y el de Antequera son hospitales comarcales en los que tiene un gran peso el seguimiento de la población residente del área mediante citas para consultas de especialistas. Sin embargo el Hospital Virgen de la Victoria es hospital de especialidades donde se debe de acudir para las urgencias de toda una serie de especialidades; más aún en el caso del Centro regional de Carlos Haya donde solo disponemos de los datos de 2.004 pero es suficiente para corroborarnos el mayor peso de las urgencias. Solo estas diferencias funcionales entre estos hospitales pueden explicar que en los comarcales se hayan incrementado más las consultas de extranjeros y en los hospitales de especialidades se hayan incrementado más la atención de urgencias de extranjeros.

Partimos pues de la base que no podemos hacer una división territorial exacta por áreas hospitalarias por tratarse de hospitales con diferentes características funcionales y por lo tanto con diferentes áreas de influencia, y además a estas diferencias funcionales se les agrega la población flotante de extranjeros en una provincia turística como es Málaga; sin embargo este mayor peso de las consultas o de las urgencias también puede de estar influido por la edad y situación de los extranjeros que residen en las zonas más cercanas al hospital. Es decir el mayor crecimiento de las consultas puede estar motivado por la mayor presencia relativa de extranjeros comunitarios tanto jubilados como activos entre los extranjeros residentes en la Costa del Sol Occidental y en la Depresión de Antequera. Frente a la creciente llegada de inmigrantes laborales no comunitarios a la capital y Valle del Guadalhorce que tienen como hospitales públicos más cercanos los ubicados en la capital, el hospital Virgen de la Victoria donde han crecido sobre todo las urgencias. Aunque solo disponemos 
de los datos del año 2004 lo corrobora el mayor peso de las urgencias en el Centro Regional de Carlos Haya también situado en la capital.

Ya podemos apuntar un primer rasgo que trataremos de comprobar y es el mayor uso de las consultas de especialistas por parte de los extranjeros comunitarios residentes debido a que proporcionalmente hay más ancianos entre ellos, mientras que los extranjeros no comunitarios al ser adultos jóvenes no precisan tanto este seguimiento sanitario y también lo eluden por sus intensas jornadas laborales acudiendo solo a la inmediata asistencia de urgencias. Para poder comprobar esta hipótesis debemos tener en cuenta la nacionalidad de los extranjeros asistidos.

Puesto que cada hospital nos han podido ofrecer diferentes tipos de datos debemos analizarlos por separado pero el conjunto resulta muy esclarecedor del uso que realizan los distintos colectivos de extranjeros de los servicios hospitalarios.

\section{EVOLUCIÓN DE LA ASISTENCIA A EXTRANJEROS POR NACIONALIDAD}

Los datos obtenidos en el Hospital Virgen de la Victoria nos permiten observar la evolución en las nacionalidades de los extranjeros asistidos desde el año 2.000 al 2.004. Para simplificar hemos agregado la información en grandes conjuntos y solo ofrecemos además individualizados los datos de aquellos países con mayor número de usuarios 


\section{CUADRO 5}

\section{EVOLUCIÓN DE LA ASISTENCIA A EXTRANJEROS EN EL H. VIRGEN DE LA VICTORIA}

\begin{tabular}{|c|c|c|c|c|c|c|c|c|c|c|c|}
\hline \multicolumn{6}{|c|}{ Asistercia a extranjeros en urgencias } & \multicolumn{6}{|c|}{ Asistencia a extranjenos cn consultas } \\
\hline & 2000 & 200 & 2002 & $2.00 \mathrm{r}$ & 2000 & & 2000 & 2007 & $20 Q$ & 2000 & 2.004 \\
\hline UF(25) & 2064 & 2304 & 2968 & 3800 & $4: 45$ & DE,25) & 2605 & 3.104 & 3.725 & $4+206$ & 5.063 \\
\hline Rends be & $2 \pi 7$ & 204 & 410 & 795 & 981 & Rruto IEr. & 145 & 257 & 356 & $4 \mathrm{Ex}$ & (S) \\
\hline Afrea & 903 & 1339 & 1663 & 2376 & $2 \times 35$ & Aftrica & 811 & 1.012 & 1312 & 1.754 & $20 t 0$ \\
\hline Aratrica & 453 & 60 & $112 x$ & 2436 & $30 \%$ & Amkirica & 451 & 523 & 800 & 1500 & 1.982 \\
\hline Nas & 232 & 271 & 308 & 351 & 45 & Asia & 161 & 189 & 254 & $3 n$ & 422 \\
\hline Rerino t'niss & 8\$3 & 925 & 1206 & $1+84$ & 1951 & Rriso t'nido & 1.168 & 1.423 & $1.7 \times 0$ & 2.164 & $2 \times 51$ \\
\hline Marrecess & 879 & 1.115 & 1383 & 1.565 & 2300 & Mamacos & 677 & 854 & 1.005 & 1.419 & 1,600 \\
\hline Argentisa & 125 & 200 & 517 & $\%$ & 1308 & Argeatina & 134 & $12 y$ & 34 & 60 & Kob \\
\hline Drscoescido & 917 & 1.142 & 1182 & 1254 & 1.101 & Descoeceliso & 138 & 67 & 74 & 71 & 105 \\
\hline TOTA. & $49 \%$ & 6019 & $7 \pi 7$ & 11091 & $12 \mathrm{CON}$ & TOTAI. & a won & 51.17 & 651 & $\mathrm{X} 581$ & 10211 \\
\hline \multicolumn{6}{|c|}{ Asulcicia a eviranjeron en ingresos } & \multicolumn{6}{|c|}{ Astucacia a cutranjeros en intervencioses } \\
\hline & 2000 & $2 \mathrm{mp}$ & 200 & $2 \mathrm{mor}$ & 2004 & & 2000 & 2007 & 2002 & 2008 & 2004 \\
\hline UE(25) & 771 & $7 \pi$ & 843 & 1000 & 1124 & DEP25) & 230 & 363 & 271 & 333 & 413 \\
\hline Besso Fer. & 34 & 43 & 69 & 57 & 84 & Resto Eser. & 14 & 20 & 3 & 42 & 56 \\
\hline Afika & 162 & 171 & 214 & 279 & 100 & Affixe & 55 & 58 & 66 & 124 & 130 \\
\hline Arstrica & 95 & 87 & $16 t$ & 280 & 318 & Amkirica & 39 & 37 & 72 & 136 & 150 \\
\hline Asa & 52 & 39 & 52 & 51 & 59 & Asia & 19 & 15 & 23 & 25 & 28 \\
\hline Recins Unibs & $39 ?$ & 373 & 427 & $\$ 65$ & $\$ 4$ & Reiss trinido & 123 & 124 & $1+6$ & 190 & 227 \\
\hline Marrecess & 133 & 131 & 164 & 210 & $2 x$ & Mamanos & 48 & 48 & 50 & 95 & 98 \\
\hline Arpentia & 17 & 22 & 41 & 80 & 9 & Arpestins & 10 & 10 & 24 & 53 & 53 \\
\hline Drocremido & 172 & 470 & did & 143 & 197 & Dewomoride & 2 & 4 & 0 & 0 & 3 \\
\hline T6э. & 1.56 & 1581 & 1.753 & $1.7 \times 0$ & $2 \times 2$ & Tor.M. & 350 & 350 & 485 & $6 \infty$ & 770 \\
\hline
\end{tabular}

Fuente: Servicio de Documentación Hospital Virgen de la Victoria. Elaboración propia

Volvemos a comprobar que en estos cinco años se han incrementado fuertemente todos los tipos de asistencia a extranjeros pero sobre todo las urgencias y las consultas que casi se han triplicado, pero además se han producido cambios en la composición por nacionalidad:

- En el año 2.000 la mayor parte de la asistencia hospitalaria la utilizaron ciudadanos comunitarios en su mayoría británicos. Solo en las urgencias acudieron los marroquíes en cantidad similar a los británicos.

- En estos años hasta 2.004 se han incrementado los extranjeros atendidos de todas las nacionalidades pero sobre todo el conjunto de americanos que aumentan su peso relativo pues en el año 2.000 significaban en torno al $10 \%$ de todos los tipos de asistencia y cuatro años después pasan a ser en torno a un 20 o un $25 \%$

- De resultas en 2.004 todavía sigue siendo mayoritaria la asistencia a extranjeros procedentes de la Unión Europea en consultas, intervenciones e ingresos, y dentro de ellos los británicos siguen siendo el grupo más numeroso. Sin embargo en las urgencias en 2.004 ya ha sido ligeramente superior la atención a ciudadanos no comunitarios por la 
fuerte asistencia de africanos y americanos, si bien por países destaca la fuerte afluencia de marroquíes y británicos.

Esta evolución coincide a grosso modo con la que se ha producido en los extranjeros residentes en estos años. Puesto que no debemos hacer una medición estadística exacta sobre intensidad de uso al tratarse de asistencias que pueden estar protagonizados por extranjeros no residentes, lo vamos a realizar de forma cualitativa y en los siguientes gráficos a la evolución en la composi-
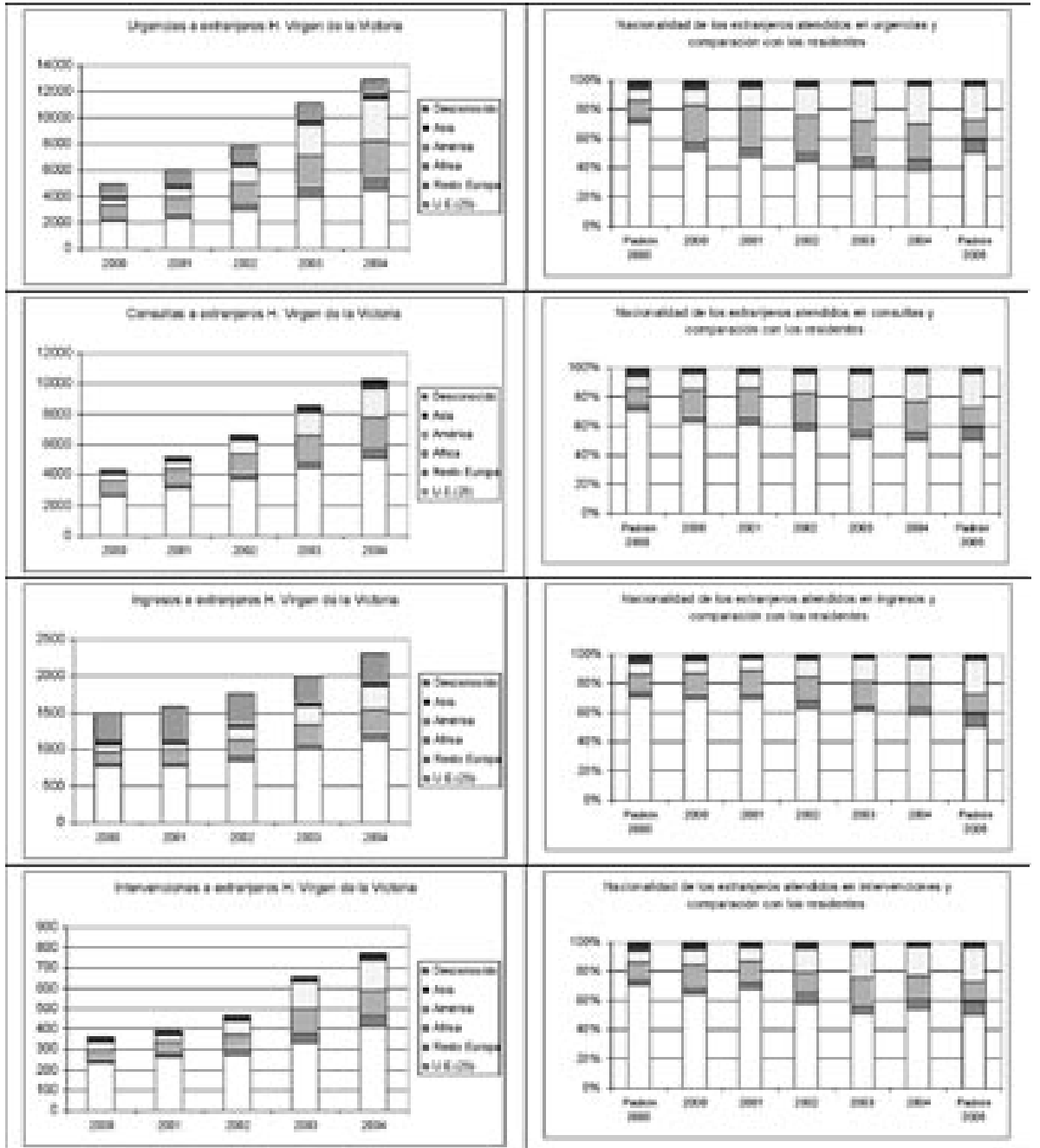

Fuente: Servicio de Documentación Hospital Virgen de la Victoria. Elaboración propia 
ción por nacionalidad de los extranjeros asistidos en el hospital durante los años 2.000 a 2.004 , le hemos agregado la composición de los extranjeros residentes según los padrones inicial y final del periodo : 1 de enero de 2.000 y de 2.005. Según el Padrón de 1 de enero de 2.000 la mayoría, un $70 \%$, de los 73.419 extranjeros residentes en la provincia de Málaga eran procedentes de la Unión Europea. En 2.005 estos comunitarios se han incrementado pero sobre todo se ha producido una fuerte llegada de ciudadanos no comunitarios de tal manera que los 181.589 extranjeros residentes en 2.005 se reparten por igual entre ciudadanos procedentes de la Unión Europea o del resto del mundo.

Si comparamos el reparto por nacionalidades de los extranjeros residentes que ha mostrado el Padrón con el reparto por nacionalidades de los diferentes tipos de asistencia aparecen diferencias:

- solo han tenido unas proporciones similares con las nacionalidades de los extranjeros residentes las consultas que en 2.004 se reparten por igual entre usuarios procedentes de la Unión Europea y del resto del mundo

- Los ingresos y las intervenciones que en el año 2.000 tuvieron un reparto por nacionalidades similar a los residentes han ido adoptando proporcionalmente un mayor uso por los ciudadanos procedentes de la Unión Europea

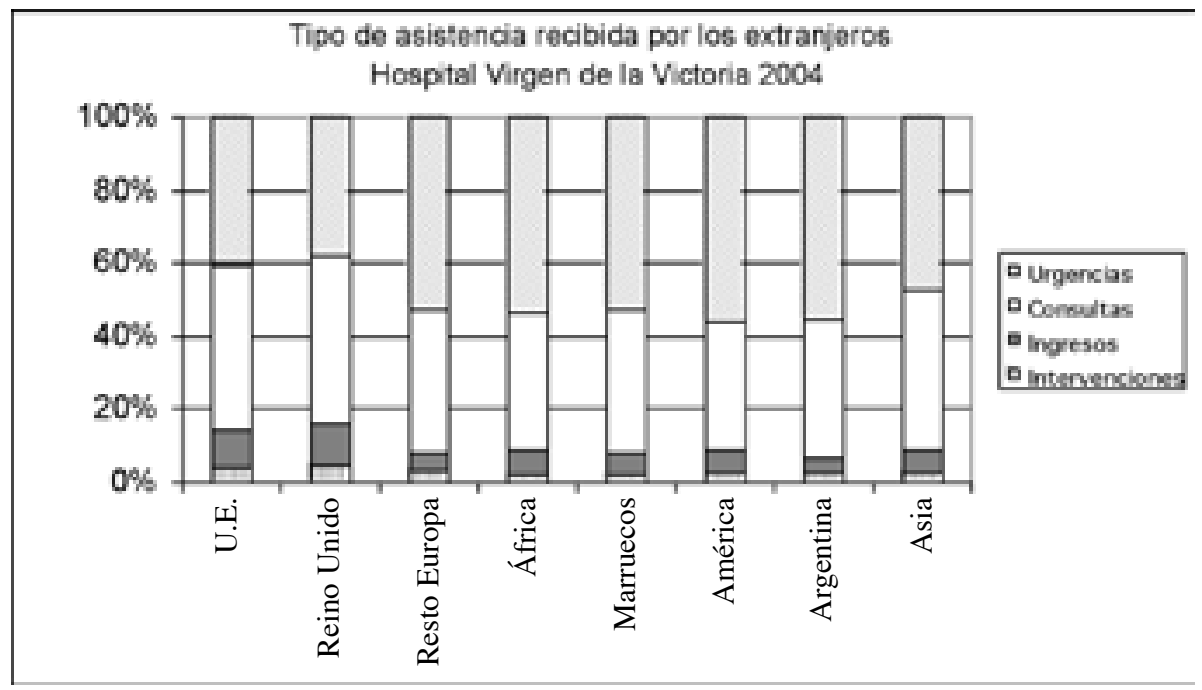

Fuente: Servicio de Documentación Hospital Virgen de la Victoria. Elaboración propia 
- Por el contrario en las urgencias ha habido y hay una mayor utilización por parte de los extranjeros no comunitarios, muy superior a la proporción de residentes.

Como resumen podemos ver el gráfico siguiente donde se señala el diferente uso de la atención hospitalaria según nacionalidad en el año 2.004: mientras los extranjeros comunitarios, entre ellos los británicos, acuden mayoritariamente para consultas, ingresos e intervenciones, todo el resto de extranjeros no comunitarios, tanto del resto de Europa, como de África, América y Asia acuden mayoritariamente a las urgencias y utilizan en menor proporción el resto de asistencias.

Como apuntamos anteriormente este mayor uso de las consultas de especialistas, de los ingresos y de las intervenciones por parte de los extranjeros comunitarios, aunque puede estar influido por otros factores, se puede explicar sencillamente por su estructura por edad debido a que proporcionalmente hay más ancianos entre ellos:

\section{CUADRO 6}

\section{ESTRUCTURA POR EDAD DE LOS EXTRANJEROS RESIDENTES EN LA PROVINCIA DE MÁLAGA (\%)}

\begin{tabular}{|l|c|c|c|c|}
\hline & Menos de 15 años & 15 a 39 & 40 a 64 & 65 y más \\
\hline Unión Europea & 11,5 & 24,4 & 40,2 & 23,9 \\
\hline Reino Unido & 12,7 & 20,3 & 42,9 & 24,0 \\
\hline Resto Europa & 10,9 & 51,1 & 31,2 & 6,7 \\
\hline África & 12,8 & 63,4 & 21,4 & 2,4 \\
\hline Marruecos & 14,4 & 60,2 & 22,8 & 2,6 \\
\hline América & 16,0 & 56,0 & 24,1 & 3,9 \\
\hline Argentina & 18,1 & 51,6 & 26,0 & 4,3 \\
\hline Asia y resto & 14,5 & 49,0 & 31,5 & 4,9 \\
\hline TOTAL & 12,8 & 40,5 & 32,7 & 14,0 \\
\hline
\end{tabular}

Fuente: Padrón de 1 de enero de 2.005. Elaboración propia.

Mientras que los extranjeros no comunitarios no han precisado tanto las consultas de especialistas, los ingresos y las intervenciones porque son mayoritariamente adultos jóvenes, a lo que se puede unir que están sometidos a intensos ritmos laborales y eluden en lo posible el seguimiento sanitario. Por estos motivos estos extranjeros no comunitarios, aunque estén empadronados y adscritos a la Seguridad Social a través del trabajo o mediante el Documento 
de reconocimiento temporal a la asistencia sanitaria, acudirán en mayor medida a la asistencia inmediata de urgencias y se les agregarán aquellos extranjeros no empadronados que carecen de derecho a la asistencia primaria pero que la ley 4/2000 les reconoce el derecho a la asistencia en urgencias.

Se ha producido también diferencias en la utilización por los extranjeros del Hospital Costa del Sol aunque en este caso solo disponemos de los datos correspondientes al año 2.003:

Asistencia a extranjeros H. Costa del Sol 2.003

\begin{tabular}{|l|c|c|c|}
\hline & Urgencias & Consultas & Ingresos \\
\hline TOTAL & 26.536 & 24.762 & 3.840 \\
\hline U.E & 11.157 & 8.837 & 1.781 \\
\hline Resto & 11.629 & 9.118 & 1.513 \\
\hline Sin datos & 3.750 & 6.807 & 546 \\
\hline R. Unido & 5.766 & 4.339 & 972 \\
\hline Marruecos & 3.250 & 1.536 & 259 \\
\hline Ecuador & 2.349 & 813 & 158 \\
\hline
\end{tabular}

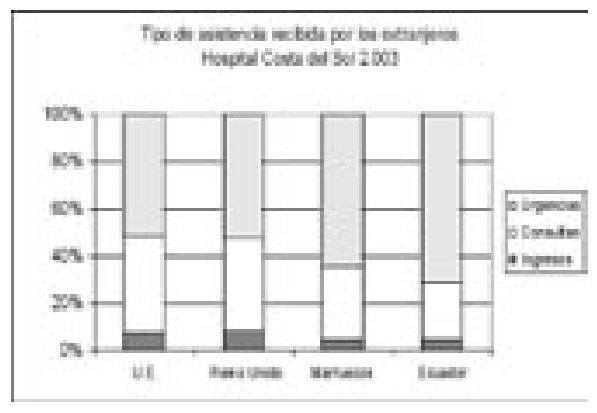

Fuente: Unidad de Evaluación. Hospital Costa del Sol. Elaboración propia

Aunque no hemos podido hacer la misma agrupación de la información si podemos observar que es muy similar el número de extranjeros asistidos pertenecientes a la Unión Europea y del resto del mundo. Pero, así como los marroquíes y ecuatorianos corroboran claramente que utilizan sobre todo las urgencias, en el caso de los extranjeros comunitarios y su nacionalidad predominante que son los ciudadanos del Reino Unido, presentan sin duda una mayor proporción de uso de las hospitalizaciones y consultas, pero utilizan también mucho las urgencias. Creemos que este uso más equilibrado de los diferentes tipos de asistencia especializada por los ciudadanos comunitarios en el Hospital Costa del Sol se debe a que se trata de una zona eminentemente turística y al fuerte uso de los extranjeros comunitarios residentes de las consultas e ingresos, se le unen los turistas que acuden solo a las urgencias. Esto lo podemos comprobar en el cuadro $\mathrm{n}^{\mathrm{o}} 7$ que recoge el lugar de residencia de los extranjeros atendidos : 


\section{CUADRO 7}

\section{LUGAR DE RESIDENCIA DE LOS EXTRANJEROS ATENDIDOS EN EL H. COSTA DEL SOL (2004)}

\begin{tabular}{|l|c|c|c|c|c|}
\hline & $\begin{array}{c}\text { Área H. } \\
\text { Costa del Sol }\end{array}$ & Resto España & Extranjero & No consta & Total \\
\hline Urgencias & 21.367 & 1.791 & 6.213 & 639 & 30.010 \\
\hline$\%$ & 71,2 & 6,0 & 20,7 & 2,1 & 100 \\
\hline Consultas & 28.532 & 1.015 & 933 & 73 & 30.553 \\
\hline$\%$ & 93,4 & 3,3 & 3,1 & 0,2 & 100 \\
\hline Ingresos & 3.503 & 247 & 532 & 10 & 4.292 \\
\hline$\%$ & 81,6 & 5,8 & 12,4 & 0,2 & 100 \\
\hline
\end{tabular}

Fuente: Unidad de Evaluación. Hospital Costa del Sol. Elaboración propia

Como es lógico en las consultas de especialistas casi la totalidad de los extranjeros asistidos residen en el área del hospital pues estas consultas se realizan básicamente para seguimiento de la población residente. Sin embargo en las urgencias más del 20\% residen en el extranjero y según podemos comprobar examinando las formas de financiación, estos usuarios que residen en el extranjero son en gran medida turistas comunitarios que están cubiertos por la Seguridad Social de su país:

\section{CUADRO 8 \\ TIPO DE FINANCIACIÓN DE LA ASISTENCIA A EXTRANJEROS EN EL H. COSTA DEL SOL (2004)}

\begin{tabular}{|l|c|c|c|c|}
\hline & Seguridad Social & Convenio U.E. (E111) & Otras & Total \\
\hline Urgencias & 17.138 & 4.751 & 8.121 & 30.010 \\
\hline$\%$ & 57,1 & 15,8 & 27,1 & 100 \\
\hline Consultas & 25.308 & 1.591 & 3.654 & 30.553 \\
\hline$\%$ & 82,8 & 5,2 & 12,0 & 100 \\
\hline Ingresos & 2.673 & 477 & 1,142 & 4.292 \\
\hline$\%$ & 62,3 & 11,1 & 26,6 & 100 \\
\hline
\end{tabular}

Fuente: Unidad de Evaluación. Hospital Costa del Sol. Elaboración propia

Podemos ver que la asistencia a extranjeros en el hospital Costa del Sol ha presentado en el caso de las consultas una cobertura mayoritaria de la Seguridad Social (82\%) como es lógico puesto que estas consultas de especia- 
listas se realizan en mayor medida para seguimiento de población residente; mientras que en las urgencias e ingresos se produce en gran proporción otras formas de financiación ( 27 y $26 \%$ ) que suelen ser medios privados de aseguradoras caso de accidentes laborales o de trafico, y al ser un espacio netamente turístico también hay una proporción importante de ciudadanos comunitarios que están cubiertos por la Seguridad Social de su país y acuden temporalmente provistos con el documento E111 (15 y 11\%).

Para terminar solo ratificar lo obvio: si los extranjeros cada vez representan una proporción mayor en el conjunto de la población lógicamente irán teniendo más peso en la atención prestada por los hospitales públicos. En el siguiente cuadro podemos comprobar que esta asistencia a extranjeros cada vez significa más en el conjunto de la actividad del hospital.

\section{CUADRO 9 \\ PORCENTAJE DE LA ASISTENCIA A EXTRANJEROS RESPECTO AL TOTAL ASISTENCIAL EN EL HOSPITAL COSTA DEL SOL}

\begin{tabular}{|c|c|c|c|}
\hline Año & Consultas & Urgencias & Ingresos \\
\hline 2001 & 10,2 & 19,6 & 18,9 \\
\hline 2002 & 11,3 & 21,9 & 21,4 \\
\hline 2003 & 12,2 & 23,0 & 22,4 \\
\hline 2004 & 13,5 & 24,5 & 24,2 \\
\hline
\end{tabular}

Fuente: Unidad de Evaluación. Hospital Costa del Sol. Elaboración propia.

Así como la llegada de extranjeros ha elevado la proporción de estos en la población pasando del 6,6\% que significaban en la provincia de Málaga en el año 2001 al 12,5\% en 2005 y en el caso del distrito sanitario de la Costa del Sol Occidental llegan a significar el 26,7\%, del mismo modo se ha incrementado su presencia en la actividad asistencial de este hospital llegando en 2004 a representar una proporción en las urgencias y en los ingresos similar a la proporción de los extranjeros en el distrito sanitario mientras que en las consultas no se llega a alcanzar dicha proporción.

\section{CONCLUSIONES}

- Todos los tipos de asistencia hospitalaria a extranjeros han crecido de 2.000 a 2.004, pero a un ritmo de crecimiento inferior al experimentado en estos años por el volumen de población extranjera residente 
- Ha crecido la asistencia hospitalaria a extranjeros y se han producido cambios en la composición por nacionalidades: en el año 2.000 la mayor parte de la asistencia hospitalaria la utilizaron ciudadanos comunitarios en su mayoría británicos. En estos años se han incrementado los extranjeros atendidos de todas las nacionalidades pero sobre todo el conjunto de americanos. De resultas en 2.004 todavía sigue siendo mayoritaria la asistencia a extranjeros procedentes de la Unión Europea en consultas, intervenciones e ingresos, y dentro de ellos los británicos siguen siendo el grupo más numeroso. Sin embargo en las urgencias en 2.004 ya ha sido ligeramente superior la atención a ciudadanos no comunitarios por la fuerte asistencia de africanos y americanos, de tal manera que los marroquíes han sido el colectivo que más han utilizado las urgencias.

- Mientras en los hospitales comarcales Costa del Sol y de Antequera el mayor crecimiento lo han experimentado las consultas de especialistas, en el hospital de especialidades Virgen de la Victoria lo han sido las urgencias. Esta diferente evolución puede obedecer a las diferencias funcionales entre estos hospitales; sin embargo este mayor peso de las consultas o de las urgencias también puede estar influido por la edad y situación de los extranjeros que residen en las zonas más cercanas al hospital.

- Los extranjeros procedentes de la Unión Europea, entre ellos los británicos que son siempre el colectivo más numeroso, utilizan en mayor medida las consultas, ingresos e intervenciones debido a que proporcionalmente hay más ancianos entre ellos. En el Hospital Costa del Sol, situado en una zona eminentemente turística, presentan una utilización algo más equilibrada porque al uso de los residentes de las consultas e intervenciones se les suman los turistas que acuden solo a las urgencias.

- Todo el resto de extranjeros no comunitarios, tanto del resto de Europa, como de África, América y Asia acuden sobre todo a las urgencias porque son mayoritariamente adultos jóvenes que no han precisado tanto las consultas de especialistas, los ingresos y las intervenciones, y además suelen estar sometidos a intensos ritmos laborales que les hace eludir en lo posible el seguimiento sanitario. Estos extranjeros no comunitarios, aunque estén empadronados y adscritos a la Seguridad Social a través del trabajo o mediante el Documento de reconocimiento temporal a la asistencia sanitaria, acudirán en mayor medida a las urgencias y se les agregarán aquellos extranjeros no empadronados que la ley les reconoce el derecho a la asistencia en urgencias. 


\section{BIBLIOGRAFÍA}

ALCALDE, R. MORENO, R. y TURBE, K. (2004): "Emigrar en la madurez, inmigrar en la vejez: las condiciones de vida de los inmigrados jubilados extranjeros en las costas mallorquinas y catalanas", $4^{\circ}$ Congreso sobre la Inmigración en España. Ciudadanía y Participación, Girona.

APARICIO, R. y TORNOS, A. (2005): El estado de bienestar y la inmigración en España, Ministerio de Trabajo.

APARICIO, L., I. y C. P. CUEVAS (2004): "El sistema de bienestar para todos. Necesidades de salud de la población inmigrante", $4^{\circ}$ Congreso sobre la Inmigración en España. Ciudadanía y Participación, Girona.

ARANGO, J. (2004): "La fisonomía de la inmigración en España“, El nuevo orden demográfico. El Campo de las Ciencias y las Artes 139, BBVA, 237-62.

BETA, C. y CAIA, M. (1998): “Consideraciones sanitarias de los británicos mayores en la Costa del Sol”, Revista Migraciones 3.

CARBALLO, M. (2005): "Migration and Maternal and Child Health: the Example of Western Europe", en International Migration and the Millenium Development Goals, UNFPA, Marrakech.

CARVAJAL, C. (2005): "Incidencia de los extranjeros en la estructura por edad de los municipios de Málaga”, Cuadernos Geográficos 36, 185-98.

CARVAJAL, C. y CORPAS, J. (2006): “Utilización de los servicios públicos sanitarios de atención primaria por los extranjeros en la provincia de Málaga”, X Congreso de la Población Española. Migraciones, movilidad y territorio, Pamplona.

CONSEJO DE EUROPA (2000): Condiciones sanitarias de los inmigrantes y refugiados en Europa, Comisión de migraciones, refugiados y demografía, Estrasburgo.

CONSEJERÍA DE GOBERNACIÓN (2004): La inmigración en Andalucía. Datos estadísticos, Junta de Andalucía.

ECHEZARRETA FERRER, M. (Dir.) (2005): El lugar europeo de retiro, Ed. Comares, Granada.

GUALDA CABALLERO, E. (2005): Hacia un "trabajo decente”: inserción sociolaboral de la población extranjera en Andalucía, Informe en prensa.

HOSPITAL COSTA DEL SOL. UNIDAD DE INVESTIGACIÓN (2003): Repercusión de los pacientes no residentes en Área Sanitaria Costa del Sol, Marbella.

INSTITUTO NACIONAL DE ESTADÍSTICA (2005): Los extranjeros residentes en España 1998-2002).

LURBE, K. (2004): "La gestión de inmigrantes extracomunitarios y refugiados como grupos de riesgo", $4^{\circ}$ Congreso sobre la Inmigración en España. Ciudadanía y Participación, Girona.

MINISTERIO DE TRABAJO (1999): Estudio sobre el coste adicional de la asistencia sanitaria de la Seguridad Social a los extranjeros, Madrid 
MORENO FUENTES, F. J. (2004): “De la igualdad a la equidad en las políticas sanitarias hacia poblaciones de origen inmigrante. Análisis comparado de los casos de Reino Unido, Francia y España”, $4^{\circ}$ Congreso sobre la Inmigración en España. Ciudadanía y Participación, Girona

PÉREZ DE COLOSÍA, M. I. (1987): “Malagueños sentenciados en el Santo Oficio de Granada de 1.550", Baetica 10, 293-307.

RODRIGUEZ, V. y WARNES, T. (2002): "Los residentes europeos mayores en España: repercusiones socioeconómicas y territoriales", El nuevo orden demográfico. El Campo de las Ciencias y las Artes 139, BBVA, 123-46. 
